\title{
The Evolving Portrait of Cancer Metastasis
}

\author{
P.B. GuPta, ${ }^{* \dagger}$ S. MANi ${ }^{\dagger}$ J. YANG ${ }^{\dagger}$ K. HARTwell, ${ }^{* \dagger}$ AND R.A. WeinBerG ${ }^{* \dagger}$ \\ *Department of Biology, Massachusetts Institute of Technology, Cambridge, Massachusetts 02142; \\ ${ }^{\dagger}$ Whitehead Institute for Biomedical Research, Cambridge, Massachusetts 02142
}

\begin{abstract}
The phenomenon of cancer metastasis remains poorly understood. We discuss here various conceptual frameworks that attempt to rationalize the mechanisms by which tumors acquire metastatic ability. Portrayal of cancer as a somatic Darwinian process occurring within a tissue fails to fully explain the phenomenon of metastatic competence. The biology of pre-neoplastic cells also complicates this picture, since the phenotypes of normal cellular precursors are clearly relevant to metastatic behavior following transformation. Recent experimental results help to shed light on these and other considerations regarding the molecular mechanisms of malignant progression.
\end{abstract}

\section{THE DARWINIAN PARADIGM OF TUMOR PROGRESSION}

Research over the past three decades has provided a solid foundation for comprehending the genetic basis of tumor formation and progression. As a consequence, we now understand that the requirement for accumulated genetic lesions underlies the progressive development of adult cancers, many of which arise over a span of decades. This multifactorial genetic requirement is reflected in the extensive genomic instability of cancer cells. Indeed, dysregulation of the cellular apparatus that ensures genomic integrity appears to be one common mechanism by which premalignant clones acquire the multiple lesions that are necessary to attain high-grade malignancy (Lengauer et al. 1997; Shih et al. 2001; Goel et al. 2003; Grady 2004; Hiyama et al. 2004).

A second mechanism by which cancer cells acquire multiple genomic lesions depends on repeated cycles of mutation and clonal selection, resulting in the sequential fixation of mutations in the bulk tumor cell population. According to this Darwinian model, tumors evolve toward greater malignancy over time as a result of the expansion of individual cell clones that have sustained novel genetic lesions conferring a selective advantage within the primary tumor microenvironment (Wolman 1983, 1986; Mertens et al. 1991; Chung et al. 1996; Mora et al. 2001). The functional contribution of specific genetic lesions to cancer pathogenesis has been established, in many cases, by associating their presence with particular cancer phenotypes using in vitro and in vivo tumor models. This Darwinian paradigm provides a compelling rationale for the presence of many of the genetic lesions commonly observed in human tumors. Examples of the selective advantage that specific lesions provide include Bcl-2 overexpression (Ehlert and Kubbutat 2001; Kirkin et al. 2004) and p53 function loss (Sidransky et al. 1992; Li et al. 1998; Ehlert and Kubbutat 2001), which confer resistance to apoptosis; $\mathrm{pRb}$ loss, which confers resistance to growth-inhibitory signals (Sage et al. 2000); and gain-of-function mutations of Ras proteins (Schleger et al. 2000; Bounacer et al. 2004), which confer the ability to proliferate in the absence of normally required mitogenic signals.

The development of distant metastases is the final step in the progression of many adult malignancies. Given its success in explaining the earlier steps of tumor progression, the Darwinian model has naturally been extended by some to encompass the ultimate phenotypes of cancer cell invasiveness and metastasis. In truth, however, the molecular mechanisms of metastasis remain poorly understood, and the experimental basis for such an extension is essentially nonexistent. Indeed, several conceptual problems and, more recently, experimental observations plague the Darwinian paradigm as it relates to the acquisition of metastatic competence.

\section{THEORETICAL DIFFICULTIES IN EXTENDING THE DARWINIAN MODEL TO CANCER METASTASIS}

The abilities to proliferate in the absence of mitogenic signals, to resist apoptotic stimuli, and to induce angiogenesis each confer a clear selective advantage on cancer cells growing within the primary tumor microenvironment. In contrast, it is hardly clear how successive cycles of selective pressure for metastatic ability to secondary organ sites (required under the Darwinian framework) would sensibly operate within the primary tumor microenvironment. Thus, it is hardly clear how the acquisition of metastatic competence by a cancer cell would result per se in its clonal expansion within a tumor. Without such an expansion, rare metastasis-competent cells would not accumulate in large numbers within a primary tumor. Given the low efficiency of the various steps of the "invasion-metastasis cascade," the likelihood of successful metastasis would be extraordinarily small.

The invasion-metastasis cascade (Stracke and Liotta 1992; Lawrence and Steeg 1996; Yoon et al. 2003) encompasses local invasiveness, intravasation into and transport through the circulation, extravasation, formation of a micrometastasis, and finally, formation of a macroscopic metastasis (this last step is sometimes referred to as 


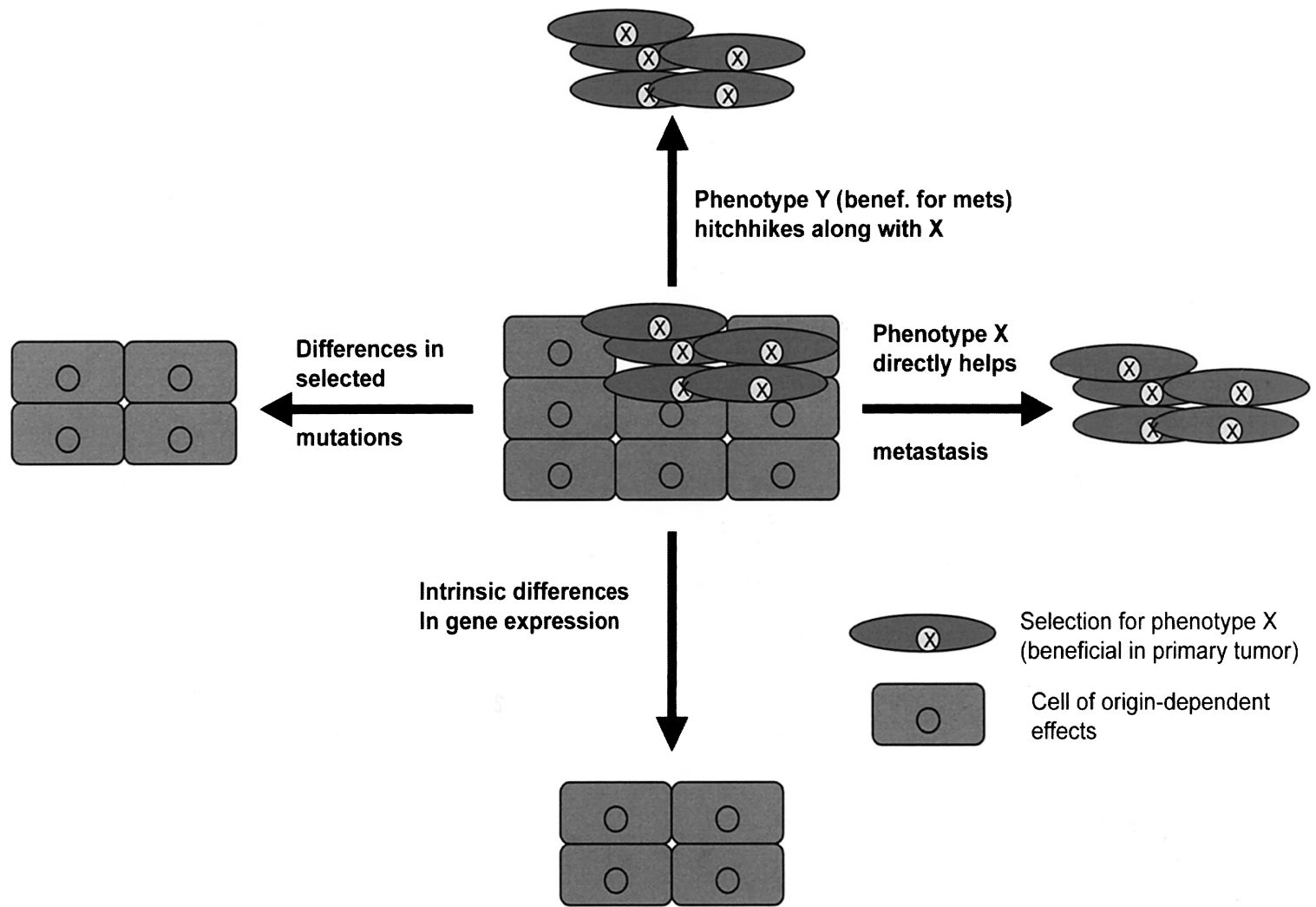

Figure 1. Schematic depiction of cancer metastasis models.

"colonization"). The complexity of this process has caused many to assume that numerous genetic lesions are required within individual cancer cells in order to enable them to complete this cascade. If this were so, the probability of any one of these mutations occurring is small, and, therefore, the likelihood of all requisite lesions occurring together within a single cell is negligibly small.

Alternative scenarios offer resolutions of this conceptual quandary (Fig. 1):

1. Some traits that are advantageous to cancer cells within the primary tumor microenvironment may also contribute to metastatic competence. Resistance to apoptosis, for example, may be dually advantageous for cancer cells - for survival within the primary tumor microenvironment and also for survival in the circulation or at secondary organ sites (prior to colonization), thereby contributing to metastatic competence.

2. Selective pressure within the primary tumor microenvironment for phenotype $\mathrm{X}$ may result in the clonal expansion of cancer cells harboring a genetic lesion that also happens to confer a second phenotype $\mathrm{Y}$, the latter contributing to metastatic competence. Thus, cancer cells may acquire a particular phenotype that is advantageous within the primary tumor microenvironment through one of several possible genetic lesions, some of which fortuitously confer additional phenotypes that facilitate cancer cell metastasis.

The possibilities delineated above describe how metastatic phenotypes may hitchhike along with pheno- types that are beneficial within the primary tumor microenvironment, resulting in the clonal expansion of metastasis-competent cells within the primary tumor. These scenarios may help to resolve the conceptual difficulties inherent in extending an evolutionary model of tumor progression to include metastasis. However, recent unanticipated observations with tumor specimens obtained from cancer patients call into question the applicability of the Darwinian framework to the acquisition of metastatic competence. In particular, classifiers based on mRNA expression profile arrays have demonstrated that the transcriptional state of relatively early-stage primary tumors is predictive of the ultimate clinical prognosis of patients (van de Vijver et al. 2002; van't Veer et al. 2002; Huang et al. 2003; Ramaswamy et al. 2003; Glinsky et al. 2004a,b; Wang et al. 2005). This surprising finding appears to be fundamentally inconsistent with the stochastic nature of metastasis implied by the Darwinian model. Thus, if it were the case that metastatic competence results from selection operating on a preexisting pool of cancer cell clones that have sustained random genomic alterations, then the ultimate metastatic competence of an early-stage tumor should not be predictable from its cohort of expressed genes. (According to the Darwinian scheme, the ultimate acquisition of metastatic competence is dependent on the nature of lesions that are randomly accumulated by the cells of a tumor late in tumor progression.) The apparent incompatibility of the Darwinian scheme with the existence of a "poor-prognosis" microarray signature remains unresolved by 1 and 2 above. 
The ability of early-stage tumor mRNA expression signatures to predict metastatic recurrence has important implications both for our conceptualization of metastasis and for the management of patient treatment. Is it possible to reconcile such observations with the Darwinian mutation/selection model of cancer metastasis? Before returning to these issues, we first turn to a discussion of the role that the cell type of origin can play in influencing tumor biology.

\section{THE INFLUENCE OF CELL TYPE OF ORIGIN ON METASTATIC PROCLIVITY}

For more than a century, oncologists have been aware that certain kinds of cancer carry a poorer clinical prognosis than other types of cancer. For instance, melanomas are notorious for rapidly progressing to invasive and metastatic states, whereas basal-cell carcinomas of the skin rarely metastasize. Empirical observations such as these indicate that the cell type from which a particular cancer arises plays a significant role in determining the likelihood that a given tumor will eventually metastasize, independently of genetic variation among individuals. The mechanistic reasons for this are obscure. As tumors become more aggressive, they tend toward greater and greater dedifferentiation. In fact, the most aggressive tumors can be dedifferentiated to a point where it is difficult or impossible to determine their tissue of origin based solely on histological examination ( $~ 5 \%$ of all cancers are diagnosed as having an unknown primary origin) (Milovic et al. 2002; Dowell 2003; Pavlidis et al. 2003). How is it, then, that malignant cancer cells retain a memory of the cell type from which they originated during later stages of tumor progression, a time at which they are often highly dedifferentiated?

There are two conceptually disparate (but not mutually exclusive) models that could serve to explain the influence of cell type of origin on the natural history and progression of cancer (Fig. 1):

3 . The cell type of origin influences metastatic competence by affecting the nature of the genetic lesions that are selected for due to their ability to confer an advantage within the primary tumor microenvironment to a cell that has acquired them. A consequence of this would be that cancers, even at an early stage of development, would have different mutational spectra, depending on their cell type of origin. This model proposes these differences in mutational spectra would be responsible for differences in metastatic propensities (perhaps due to mechanisms 1 or 2 described in the previous subsection).

4. The expression, at various stages of tumor progression, of particular proteins that can influence eventual metastatic competence is in part dependent on the initial differentiation state of a cell prior to neoplastic conversion. Stated differently, the initial differentiation state of a normal founder cell, operating in conjunction with its spectrum of acquired mutations, exerts a strong influence on the eventual expression of metastasis-related proteins in the neoplastic descendants of this founder cell.

Currently available experimental data do not suffice to establish or exclude the importance of either of these mechanisms to human cancer. On the one hand, it is apparent from various genomic profiling studies that certain types of cancers are more prone to possess particular genetic lesions; for example, N-ras mutations or PTEN deletions, providing support for model 3. Experimental evidence in support of model 4 is much harder to come by. Later in this paper, we discuss experiments from our own research group and from other laboratories addressing the plausibility of model 4 .

\section{THE RELEVANCE OF CELL TYPE OF ORIGIN TO THE INTERPRETATION OF RECENT TUMOR MICROARRAY DATA}

Classifiers based on microarray profiles are readily able to distinguish between different types of cancers and, in some cases, afford a finer distinction between cancer subtypes than is achievable using visual pathological examination alone (Golub et al. 1999; Ramaswamy et al. 2001; Nutt et al. 2003). One can, for example, imagine a situation where the bioinformaticist is supplied with a set of 100 gene expression profiles comprising 50 basal cell carcinomas and 50 melanomas of the skin, all derived from early-stage tumors. One can further envision that the bioinformaticist is given these 100 profiles in a blinded fashion in the absence of any information about the pathological classification of the tumor samples from which they derive, being supplied information only about the clinical outcome of the patient with respect to metastatic recurrence. Having analyzed these data, the bioinformaticist can readily distinguish two subclasses of skin cancer and will be in a position to note that one profile subclass (corresponding to melanomas) affords a significantly poorer prognosis than the other profile subclass (corresponding to basal cell carcinomas). Hence, the bioinformaticist will have constructed classifiers based on gene expression profiles of early-stage tumors that can predict the likelihood of eventual metastatic recurrence and will have done so without knowledge a priori of the intrinsic subclasses into which the tumors segregate.

Similarly, it may be that tumors currently classified as identical on the basis of visual pathological examination alone may be found to harbor subclasses that originate from different cell types and exhibit distinct clinical outcomes, including progression to a metastatic state. If this were indeed the case, then the gene expression analyses would be identifying differences in the cell type of origin, which would in turn underlie differences in both the gene expression profiles and metastatic proclivity. Assuming this scenario, the possibilities 3 and 4 delineated above would have the following respective correlates:

5. Two types of early-stage tumors that appear phenotypically similar may in fact harbor very different sets of genetic lesions. One set of tumors might possess lesions that are permissive of future metastasis-enabling 
lesions. A second set of early-stage tumors might possess lesions that, for any number of reasons, are not permissive for additional metastasis-enabling mutations. Gene expression analyses may be able to distinguish between these two types of tumors, which, despite phenotypic similarity, sustain very different genetic alterations and represent different tumor subclasses.

6. Specific proteins that can contribute at some point to metastatic competence may be expressed intensely in some tumors and not in others, depending on the cell type from which the cancer originated. The expression of the aforementioned proteins may not be sufficient to confer metastatic competence, but may collaborate with other proteins to program metastatic ability, perhaps at later stages of tumor progression. Gene expression analyses would be able to distinguish between these two subclasses of tumors.

\section{CAN PREEXISTING CELLULAR STATES INFLUENCE INVASIVE OR METASTATIC BEHAVIOR?}

As we have noted in the discussion above, empirical observations suggest that differences in preexisting epigenetic or transcriptional states can provide contributions to cancer cell phenotypes in a manner that is not fully attributable to differences in genetic lesions alone. The question remains as to whether there is experimental evidence to support this notion, particularly with respect to cellular phenotypes that are relevant for metastatic dissemination. Below, we discuss two sets of experimental observations in which epigenetically programmed cellular states provide significant contributions to invasive and metastatic behaviors.

\section{EMTS IN DEVELOPMENT}

An important epigenetic program that operates during a variety of morphogenetic processes in early embryogenesis closely resembles the behavior of cancer cells that have progressed to a highly malignant state. This raises the question of whether, during malignant progression, some of these embryonic cell biological programs are resurrected by cancer cells in order to acquire the multiple traits associated with high-grade malignancy, a notion that we explore later. During embryogenesis, subpopulations of cells undergo profound changes in morphology and motility. For example, initiation of gastrulation occurs with the invagination of the ectoderm through the blastopore and subsequent cellular migration along defined tracts within the embryo, resulting in eventual mesoderm specification. As another example, cells of the vertebrate neural crest lineage, which is specified along the dorso-lateral side of the notochord, are characterized by extensive migration throughout the embryo, ultimately giving rise to several adult cell types. In the late 1800 s, embryologists observed that these and other developmental migrations were often associated with specific changes in cellular morphology. In particular, they noted that embryonic cells which previously possessed epithelial qualities lost their characteristic polarity, cell-cell junctions, and cytoskeletal components prior to migration. This process was later dubbed an epithelial-tomesenchymal transition (EMT) (Thiery 2003).

\section{MOLECULAR MEDIATORS OF EMT}

What are the physiological stimuli that induce EMTs during development? Embryological tissue engraftment studies have indicated that the initial impetus for a cell to undergo an EMT originates from extracellular cues, e.g, other nearby cells. Molecular and genetic studies have indicated that TGF- $\beta$ and Wnt family proteins are important recurrent players in mediating many of the various EMTs that occur during development (Oft et al. 1998; Thiery 2003; Kemler et al. 2004). Also important are HGF and FGF-1, which induce epithelial cell scattering without the concomitant up-regulation of mesenchymal marker (one might call these "partial EMTs") (Thiery and Chopin 1999).

Many of these extracellular signals induce the expression of transcription factors that are prominent regulators of EMT (Martinez-Alvarez et al. 2004; Savagner et al. 2005). These include members of the Snail superfamily of zinc finger proteins (e.g., Snail, Slug, Escargot), as well as bHLH proteins (e.g., Twist, Sip1). In some cases, these factors have been shown to directly repress transcription of the E-cadherin gene, a key epithelial protein. However, little is known about other functional contributions that these transcription factors may provide to EMT, and this remains an important area for future research.

\section{EMT AND CANCER CELL INVASION AND METASTASIS}

As a general rule, EMTs are not observed in the adult organism following their transient induction in development. Two notable exceptions include the healing of epithelial injury (where EMT occurs in the cells lining the wound tract) (Tanaka et al. 2004; Savagner et al. 2005) and organs in which there is significant postnatal epithelial development. Accordingly, it is not surprising that the expression of extracellular proteins and transcription factors that can induce EMT is tightly regulated under normal conditions in adult epithelia. However, many factors that induce EMT in development are aberrantly expressed by cancer epithelial cells during tumorigenesis and are implicated in cancer invasion and progression. To name just a few, these include HGF, TGF- $\beta$, Rho family proteins, Snail, Slug, and Twist. This observation, together with the association of EMTs with invasive behaviors during development, has naturally led to the proposal that EMTs may play an important role in cancer cell invasion. Experiments in culture have confirmed that the experimental induction of EMT in cancer epithelial cells can enhance their motility and invasiveness using in vitro assays. However, the notion that EMT contributes to cancer cell invasion in vivo has yet to gain widespread acceptance, in large part due to the difficulty in establishing the bona fide occurrence of EMT in human carcinomas.

We note that the current debate over the relevance of the EMT to cancer invasion is reminiscent of a similar de- 
bate that raged 5-10 years ago regarding the significance of cellular senescence for tumor suppression (Dimri and Campisi 1994; Wynford-Thomas 1999). At that time, experiments in culture had established that key growth-inhibitory signaling proteins such as p53, pRb, p14ARF, and p16INK4a were clearly relevant to both cellular senescence and tumor formation; however, a convincing demonstration that senescence occurs in vivo was not forthcoming. To a large extent, this difficulty was a direct consequence of the lack of credible and specific functional markers for cellular senescence in vivo ( $\beta$-galactosidase activity at low $\mathrm{pH}$, although in common use, was of questionable functional relevance). Indeed, the principal criterion for gauging senescence in vitro is morphological and cannot be readily translated to an in vivo setting (Harley 1991; Urquidi et al. 2000). The resolution of this debate proceeded from the recognition that, regardless of whether cellular senescence occurs in vivo, the established overlap between tumor-suppressor pathways and pathways governing the onset of senescence ensured the relevance of this phenomenon to cancer genetics.

Many of these characteristics are shared by the EMT phenomenon: (1) An EMT is most readily observed in pure populations of epithelial cells in culture on the basis of morphologic and motility alterations; (2) an EMT is not readily identifiable in vivo, due to the nature of the criterion just described together with the difficulty in distinguishing epithelial cells that have undergone an EMT from nearby bona fide mesenchymal cells in the tumor stroma; (3) there is a significant overlap between molecular pathways controlling the invasive and migratory behaviors of cancer cells and the pathways governing the induction of EMT. Similar to the situation with cellular senescence, we believe that the overlap between signal transduction pathways mediating EMT and pathways involved in cancer cell invasiveness ensures its relevance to the biology of cancer.

How does all this affect the previous discussion pertaining to experiments indicating that gene expression data can predict invasive behavior? EMT is the prime example of an epigenetically programmed "cellular differentiation state" that (1) does not require genetic alterations for its induction or propagation, (2) is discernible using gene expression profile-based classifiers, and (3) can significantly influence the invasive and migratory properties of normal and carcinomatous epithelial cells. In this respect, the EMT is an example of an experimental phenomenon supporting the plausibility of model 4 described above. In particular, the finding that cancer cells which have undergone an EMT can display increased invasive behaviors demonstrates the plausibility of the notion that cellular differentiation states can affect cancer cell invasiveness in a significant manner independently of genomic lesions.

\section{MELANOMA AND METASTASIS}

A second experimental observation supporting model 5 involves the neoplastic transformation of primary human cell types via the introduction in vitro of a defined series of genetic elements. This system utilizes the Simian Virus 40 early region (SV40ER), which encodes the viral large $\mathrm{T}$ (LT) and small T (st) oncoproteins, in conjunction with a gene encoding the catalytic subunit of the telomerase holoenzyme (hTERT) to immortalize primary human cells; the resulting immortalized cells are then transformed by introducing a ras oncogene. A variety of human cell types transformed in this manner generate localized tumors that have minimal ability to metastasize (Hahn et al. 1999; Elenbaas et al. 2001; Rich et al. 2001; Lundberg et al. 2002). A surprising exception is observed with primary human melanocytes, which exhibit a highly penetrant metastatic phenotype following transformation (Gupta et al. 2005). Because the above-described procedure generates cancer cell lines transformed with an identical set of genes, variations in tumor phenotypes arising from genetic differences between cancer cell lines are effectively minimized. In particular, the uniquely metastatic behavior of transformed melanocytes in this system is most likely a consequence of their differentiation program and is, conversely, not due to differences in genetic lesions.

These observations suggested that intrinsic factors associated with the melanocyte differentiation program can provide a significant contribution to melanoma's metastatic ability. In fact, the neural crest cell factor, Slug, is expressed both in the transformed melanocytes and in human melanocytic nevi in situ (Gupta et al. 2005). This transcription factor, which is known to be required for neural crest cell migration during development (Cheung et al. 2005), provided an important functional contribution to the metastatic ability of the experimentally generated melanoma cells. Since dermal melanocytes are derived from the neural crest, these findings raise the intriguing possibility that factors mediating migratory behaviors in particular embryonic cell types might also contribute to invasiveness following transformation of their differentiated progeny in adults.

\section{CONCLUSION}

Recent experimental observations have challenged the conventional conceptual underpinnings of cancer metastasis. Although the genetic heterogeneity-clonal selection model remains indispensable for comprehending malignant progression, it appears likely that other molecular and cellular processes are also relevant to the phenomenon of cancer metastasis. A better understanding of the contribution that the originating cell type of a cancer provides to its metastatic potential may be helpful in interpreting the prognosis-prediction ability of tumor gene expression profiling data. A further examination of the interplay between differentiation state, neoplastic transformation, and metastasis is an important area for future investigation and has broad ramifications for our understanding of metastasis.

\section{ACKNOWLEDGMENTS}

This work was supported by U.S. Army Pre-doctoral Breast Cancer Fellowship DAMD17-02-1-0468 (P.B.G.) and by National Institutes of Health/National Cancer Institute grant RO1CA78461-05 (R.A.W.). R.A.W. is an 
American Cancer Society Research Professor and a Daniel K. Ludwig Foundation Cancer Research Professor.

\section{REFERENCES}

Bounacer A., McGregor A., Skinner J., Bond J., Poghosyan Z., and Wynford-Thomas D. 2004. Mutant ras-induced proliferation of human thyroid epithelial cells requires three effector pathways. Oncogene 23: 7839.

Cheung M., Chaboissier M.C., Mynett A., Hirst E., Schedl A., and Briscoe J. 2005. The transcriptional control of trunk neural crest induction, survival, and delamination. Dev. Cell 8: 179.

Chung G.T., Sundaresan V., Hasleton P., Rudd R., Taylor R., and Rabbitts P.H. 1996. Clonal evolution of lung tumors. Cancer Res. 56: 1609.

Dimri G.P. and Campisi J. 1994. Molecular and cell biology of replicative senescence. Cold Spring Harbor Symp.Quant. Biol. 59: 67.

Dowell J.E. 2003. Cancer from an unknown primary site. Am. J. Med. Sci. 326: 35.

Ehlert J.E. and Kubbutat M.H. 2001. Apoptosis and its relevance in cancer therapy. Onkologie 24: 433.

Elenbaas B., Spirio L., Koerner F., Fleming M.D., Zimonjic D.B., Donaher J.L., Popescu N.C., Hahn W.C., and Weinberg R.A. 2001. Human breast cancer cells generated by oncogenic transformation of primary mammary epithelial cells. Genes Dev. 15: 50.

Glinsky G.V., Higashiyama T., and Glinskii A.B. 2004a. Classification of human breast cancer using gene expression profiling as a component of the survival predictor algorithm. Clin. Cancer Res. 10: 2272.

Glinsky G.V., Glinskii A.B., Stephenson A.J., Hoffman R.M., and Gerald W.L. 2004b. Gene expression profiling predicts clinical outcome of prostate cancer. J. Clin. Invest. 113: 913.

Goel A., Arnold C.N., Niedzwiecki D., Chang D.K., Ricciardiello L., Carethers J.M., Dowell J.M., Wasserman L., Compton C., Mayer R.J., Bertagnolli M.M., and Boland C.R. 2003. Characterization of sporadic colon cancer by patterns of genomic instability. Cancer Res. 63: 1608.

Golub T.R., Slonim D.K., Tamayo P., Huard C., Gaasenbeek M., Mesirov J.P., Coller H., Loh M.L., Downing J.R., Caligiuri M.A., Bloomfield C.D., and Landes E.S. 1999. Molecular classification of cancer: Class discovery and class prediction by gene expression monitoring. Science 286: 531.

Grady W.M. 2004. Genomic instability and colon cancer. Cancer Metastasis Rev. 23: 11

Gupta P.B., Kuperwasser C., Brunet J.P., Ramaswamy S., Kuo W.L., Gray J.W., Naber S.P., and Weinberg R.A. 2005. The melanocyte differentiation program predisposes to metastasis after neoplastic transformation. Nat. Genet. 37: 1047.

Hahn W.C., Counter C.M., Lundberg A.S., Beijersbergen R.L., Brooks M.W., and Weinberg R.A. 1999. Creation of human tumour cells with defined genetic elements. Nature 400: 464.

Harley C.B. 1991. Telomere loss: Mitotic clock or genetic time bomb? Mutat. Res. 256: 271.

Hiyama T., Tanaka S., Yoshihara M., Sasao S., Kose K., Shima H., Tuncel H., Ueno Y., Ito M., Kitadai Y., Yasui W., Haruma K., and Chayama K. 2004. Chromosomal and microsatellite instability in sporadic gastric cancer. J. Gastroenterol. Hepatol. 19: 756.

Huang E., Cheng S.H., Dressman H., Pittman J., Tsou M.H., Horng C.F., Bild A., Iversen E.S., Liao M., Chen C.M., West M., Nevins J.R., and Huang A.T. 2003. Gene expression predictors of breast cancer outcomes. Lancet 361: 1590.

Kemler R., Hierholzer A., Kanzler B., Kuppig S., Hansen K., Taketo M.M., de Vries W.N., Knowles B.B., and Solter D. 2004. Stabilization of beta-catenin in the mouse zygote leads to premature epithelial-mesenchymal transition in the epiblast. Development 131: 5817.

Kirkin V., Joos S., and Zornig M. 2004. The role of Bcl-2 family members in tumorigenesis. Biochim. Biophys. Acta 1644: 229.
Lawrence J.A. and Steeg P.S. 1996. Mechanisms of tumor invasion and metastasis. World J. Urol. 14: 124.

Lengauer C., Kinzler K.W., and Vogelstein B. 1997. Genetic instability in colorectal cancers. Nature 386: 623.

Li G., Tang L., Zhou X., Tron V., and Ho V. 1998. Chemotherapy-induced apoptosis in melanoma cells is p53 dependent. Melanoma Res. 8: 17.

Lundberg A.S., Randell S.H., Stewart S.A., Elenbaas B., Hartwell K.A., Brooks M.W., Fleming M.D., Olsen J.C., Miller S.W., Weinberg R.A., and Hahn W.C. 2002. Immortalization and transformation of primary human airway epithelial cells by gene transfer. Oncogene 21: 4577.

Martinez-Alvarez C., Blanco M.J., Perez R., Rabadan M.A., Aparicio M., Resel E., Martinez T., and Nieto M.A. 2004. Snail family members and cell survival in physiological and pathological cleft palates. Dev. Biol. 265: 207.

Mertens F., Heim S., Mandahl N., Johansson B., Mertens O., Persson B., Salemark L., Wennerberg J., Jonsson N., and Mitelman F. 1991. Cytogenetic analysis of 33 basal cell carcinomas. Cancer Res. 51: 954.

Milovic M., Popov I., and Jelic S. 2002. Tumor markers in metastatic disease from cancer of unknown primary origin. Med. Sci. Monit. 8: MT25.

Mora J., Cheung N.K., and Gerald W.L. 2001. Genetic heterogeneity and clonal evolution in neuroblastoma. Br. J. Cancer 85: 182 .

Nutt C.L., Mani D.R., Betensky R.A., Tamayo P., Cairncross J.G., Ladd C., Pohl U., Hartmann C., McLaughlin M.E., Batchelor T.T., Black P.M., von Deimling A., Pomeroy S.L., Golub T.R., and Louis D.N. 2003. Gene expression-based classification of malignant gliomas correlates better with survival than histological classification. Cancer Res. 63: 1602.

Oft M., Heider K.H., and Beug H. 1998. TGFbeta signaling is necessary for carcinoma cell invasiveness and metastasis. Curr. Biol. 8: 1243.

Pavlidis N., Briasoulis E., Hainsworth J., and Greco F.A. 2003. Diagnostic and therapeutic management of cancer of an unknown primary. Eur. J. Cancer 39: 1990.

Ramaswamy S., Ross K.N., Lander E.S., and Golub T.R. 2003. A molecular signature of metastasis in primary solid tumors. Nat. Genet. 33: 49.

Ramaswamy S., Tamayo P., Rifkin R., Mukherjee S., Yeang C.H., Angelo M., Ladd C., Reich M., Latulippe E., Mesirov J.P. Poggio T., Gerald W., Loda M., Lander E.S., and Golub T.R. 2001. Multiclass cancer diagnosis using tumor gene expression signatures. Proc. Natl. Acad. Sci. 98: 15149.

Rich J.N., Guo C., McLendon R.E., Bigner D.D., Wang X.F., and Counter C.M. 2001. A genetically tractable model of human glioma formation. Cancer Res. 61: 3556.

Sage J., Mulligan G.J., Attardi L.D., Miller A., Chen S., Williams B., Theodorou E., and Jacks T. 2000. Targeted disruption of the three Rb-related genes leads to loss of $\mathrm{G}(1)$ control and immortalization. Genes Dev. 14: 3037.

Savagner P., Kusewitt D.F., Carver E.A., Magnino F., Choi C., Gridley T., and Hudson L.G. 2005. Developmental transcription factor slug is required for effective re-epithelialization by adult keratinocytes. J. Cell. Physiol. 202: 858.

Schleger C., Heck R., and Steinberg P. 2000. The role of wildtype and mutated N-ras in the malignant transformation of liver cells. Mol. Carcinog. 28: 31.

Shih I.M., Zhou W., Goodman S.N., Lengauer C., Kinzler K.W., and Vogelstein B. 2001. Evidence that genetic instability occurs at an early stage of colorectal tumorigenesis. Cancer Res. 61: 818 .

Sidransky D., Mikkelsen T., Schwechheimer K., Rosenblum M.L., Cavanee W., and Vogelstein B. 1992. Clonal expansion of p53 mutant cells is associated with brain tumour progression. Nature 355: 846.

Stracke M.L. and Liotta L.A. 1992. Multi-step cascade of tumor cell metastasis. In Vivo 6: 309.

Tanaka T., Saika S., Ohnishi Y., Ooshima A., McAvoy J.W., Liu C.Y., Azhar M., Doetschman T., and Kao W.W. 2004. Fibroblast growth factor 2: Roles of regulation of lens cell proliferation and epithelial-mesenchymal transition in response 


\section{CANCER METASTASIS}

to injury. Mol. Vis. 10: 462 .

Thiery J.P. 2003. Epithelial-mesenchymal transitions in development and pathologies. Curr. Opin. Cell Biol. 15: 740.

Thiery J.P. and Chopin D. 1999. Epithelial cell plasticity in development and tumor progression. Cancer Metastasis Rev. 18: 31 .

Urquidi V., Tarin D., and Goodison S. 2000. Role of telomerase in cell senescence and oncogenesis. Annu. Rev. Med. 51: 65.

van de Vijver M.J., He Y.D., van 't Veer L.J., Dai H., Hart A.A., Voskuil D.W., Schreiber G.J., Peterse H.L., Roberts C., Marton M.J., Parrish M., Atsma D., Witteveen A.T., Glas A., Delahaye L., van der Velde T., Bartelink H., Rodenhuis S., Rutgers E.T., Friend S.H., and Bernards R. 2002. A gene-expression signature as a predictor of survival in breast cancer. N. Engl. J. Med. 347: 1999.

van 't Veer L.J., Dai H., van de Vijver M.J., He Y.D., Hart A.A., Mao M., Peterse H.L., van der Kooy K., Marton M.J., Witteveen A.T., Schreiber G.J., Kerkhoven R.M., Roberts C.,
Linsley P.S., Bernards R., and Friend S.H. 2002. Gene expression profiling predicts clinical outcome of breast cancer. Nature 415: 530.

Wang Y., Klijn J.G., Zhang Y., Sieuwerts A.M., Look M.P., Yang F., Talantov D., Timmermans M., Meijer-van Gelder M.E., Yu J., Jatkoe T., Berns E.M., Atkins D., and Foekens J.A. 2005. Gene-expression profiles to predict distant metastasis of lymph-node-negative primary breast cancer. Lancet 365: 671 .

Wolman S.R. 1983. Karyotypic progression in human tumors. Cancer Metastasis Rev. 2: 257.

. 1986. Cytogenetic heterogeneity: Its role in tumor evolution. Cancer Genet. Cytogenet. 19: 129.

Wynford-Thomas D. 1999. Cellular senescence and cancer. $J$. Pathol. 187: 100.

Yoon S.O., Park S.J., Yun C.H. and Chung A.S. 2003. Roles of matrix metalloproteinases in tumor metastasis and angiogenesis. J. Biochem. Mol. Biol. 36: 128. 


\section{$8_{8}^{\infty} \mathrm{CSH} \&$ Cold Spring Harbor Symposia SYMPOSIA on Quantitative Biology}

\section{The Evolving Portrait of Cancer Metastasis}

P.B. GUPTA, S. MANI, J. YANG, et al.

Cold Spring Harb Symp Quant Biol 2005 70: 291-297

Access the most recent version at doi:10.1101/sqb.2005.70.033

References This article cites 50 articles, 13 of which can be accessed free at: http://symposium.cshlp.org/content/70/291.full.html\#ref-list-1

\section{License}

Email Alerting Receive free email alerts when new articles cite this article - sign up in Service the box at the top right corner of the article or click here. 